Pacific Journal of Mathematic 


\title{
RAMSEY QUANTIFIERS AND THE FINITE COVER PROPERTY
}

\author{
John T. BALdWIN AND DAVID W. Kueker
}

\begin{abstract}
The main results of this paper are the following Theorem A. The Magidor-Malitz quantifier (in the $\boldsymbol{\aleph}_{0}$-interpretation) is eliminable from a countable stable theory $T$ if and only if $T$ does not have the finite cover property. Theorem $B$. There is an $\boldsymbol{N}_{0}$-categorical theory which is not finitely model complete.
\end{abstract}

We consider various extensions of a countable first-order logic obtained by adding $n$-ary quantifiers which assert the existence of of a "large" set of $n$-tuples satisfying the formula following the quantifier. Specifically, the Ramsey (or Magidor-Malitz) quantifier $Q^{n}$ is defined in the $\boldsymbol{W}_{0}$-interpretation by $A \vDash{ }_{0} Q^{n} x_{1}, \cdots, x_{n} \varphi\left(x_{1}, \cdots, x_{n}, \bar{a}\right)$ iff there is an infinite $Y \subset A$ which is homogeneous for $\varphi$, i.e., such that $A \vDash_{0} \varphi\left(y_{1}, \cdots, y_{n}, \bar{a}\right)$ for all $\left\langle y_{1}, \cdots, y_{n}\right\rangle \in Y^{n}$. We denote by $L^{*}$ the language which adjoins all the quantifiers $Q^{n}$ and by $L_{0}^{*}$ the associated logic in the $\boldsymbol{\aleph}_{0}$-interpretation. Our principal concern is to identify those complete first order theories $T$ in $L$ which remain complete as theories of $L_{0}^{*}$. A sufficient condition on $T$ is

$E_{0}$ : For every formula $\varphi$ in $L_{0}^{*}$, there is a formula $\psi$ in $L$ such that $T \vDash_{0} \varphi \leftrightarrow \psi$ (i.e., if $A \vDash_{0} T$ then $A \vDash_{0} \varphi \leftrightarrow \psi$ ).

If $E_{0}$ holds, we say $Q$ is eliminable in $T$.

Earlier Vinner [16] has shown that for an $\aleph_{1}$-categorical theory, the quantifier "there exists infinitely many" is eliminable. Winkler [17] showed the eliminability of the quantifier "there are infinitely many sequences" in an $\aleph_{1}$-categorical theory. It is easy to see that either of these is eliminable in an $\boldsymbol{\aleph}_{0}$-categorical theory. Cowles [4] showed that the Ramsey quantifier in the $\aleph_{0}$-interpretation was eliminable from the theory of algebraically closed fields. Keisler [8] introduced the notion of the finite cover property (f.c.p.) and showed that every $\aleph_{1}$-categorical theory fails to have the f.c.p. We generalize these elimination results by showing that if $T$ does not have the f.c.p. then all the above quantifiers are eliminable from $T$. Moreover, we characterize those stable theories $T$ which do not have the f.c.p. as exactly those which satisfy $E_{0}$. We also show that if $T$ is $\aleph_{1}$-categorical then $T$ admits elimination of these quantifiers in the equi-cardinal interpretation (again generalizing Cowles [4]).

It is natural to ask whether there is some sort of "first order property" of $T$ which is equivalent to $E_{0}$. We show several candi- 
dates for such a condition do not work. One of the examples adduced for this purpose answers a question of Paillet [10]; it is $\aleph_{0}$-categorica\} but not finitely model complete.

We first observe $E_{0}$ is equivalent to a somewhat more concrete condition. For any formula $\varphi\left(x_{1}, \cdots, x_{m}, \bar{v}\right)$ of $L$ there is a first order formula $H_{x}^{m} \bar{x} \varphi(x, v)$ which holds iff there is a set of cardinality $\geqq n$ which is homogeneous for $\varphi$. (We will omit the superscript $m$ in the future.) Now the concrete condition is

\section{$A_{0}:$ For every $L$-formula $\varphi(\bar{x}, \bar{v})$ there is an $n$ such that $T \models_{0} \forall \bar{v}\left(H_{n} \bar{x} \varphi(\bar{x}, \bar{v}) \rightarrow Q \bar{x} \varphi(\bar{x}, \bar{v})\right)$.}

THEOREM $1 . \quad E_{0} \Leftrightarrow A_{0}$.

Proof. $A_{0}$ implies $E_{0}$ by induction on formulas of $L_{0}^{*}$. For the converse suppose $E_{0}$ but that $A_{0}$ fails for the formula $\varphi(\bar{x}, \bar{v})$. By $E_{0}$ there is a $\psi(\bar{v})$ of $L$ such that $T \models_{0} Q \bar{x} \varphi(\bar{x}, \bar{v}) \Leftrightarrow \psi(\bar{v})$. Add to $L$ a unary predicate symbol $U$ and constants $\bar{c}$. Since $A_{0}$ fails, by compactness, the following set $\Gamma$ of sentences is consistent:

$$
T \cup\left\{\forall x_{1}, \cdots, \forall x_{n}\left[\Lambda_{1}^{n} U\left(x_{i}\right) \longrightarrow \varphi(\bar{x}, \bar{c})\right], \sim \psi(\bar{c})\right\} \cup\left\{\exists^{\geqq n} x U(x): n \in \omega\right\} .
$$

But any model of $\Gamma$ contradicts $E_{0}$.

The next result was observed independently by Baudisch [2].

THEOREM 2. If $T$ is $\aleph_{0}$-categorical then $E_{0}$ holds of $T$.

Proof. We show $A_{0}$ holds. For any formula $\varphi(\bar{x}, \bar{v})$, by the RyllNardjewski theorem only finitely many of the formulas $H_{n} \bar{x} \varphi(\bar{x}, \bar{v})$ can be inequivalent. Hence for some $N, T \vDash \forall x\left(H_{N} \bar{x} \varphi(\bar{x}, \bar{v}) \rightarrow H_{k} \bar{x} \varphi(\bar{x}, \bar{v})\right)$ for all $k \geqq N$. If $T \vDash_{0} H_{N} \bar{x} \varphi(\bar{x}, \bar{v}) \rightarrow Q \bar{x} \varphi(\bar{x}, \bar{v})$ then for some $\bar{a} \in A$. the countable model of $T, A \models_{0} H_{N} \bar{x} \varphi(\bar{x}, \bar{a}) \wedge \sim Q \bar{x} \varphi(\bar{x}, \bar{a})$. Let $\psi(\bar{v})$ generate the principal type realized by $\bar{a}$. Then by a compactness argument as in Theorem 1 , and $\boldsymbol{\aleph}_{0}$-categoricity of $T$, there is a $\bar{b}$ in $A$ such that $A \models_{0} \psi(\bar{b}) \wedge Q \bar{x} \varphi(\bar{x}, \bar{b})$. But this contradicts the homogeneity of $A$.

We cannot improve this by assuming that $T$ has only finitely many countable models since the Ehrenfeucht example $T$ of a theory with 3 countable models (in its finite language version [15]) does not satisfy $E_{0}$. This example is connected to our later results since the archetypal example of an $\omega$-stable theory with the f.c.p. (an equivalence relation with one class of each finite cardinality) is interpretable in $T$.

We now want to show that if $T$ does not have the f.c.p. then $T$ 
satisfies $E_{0}$. Before dealing directly with the f.c.p. we introduce an intermediate condition $M$ on the existence of maximal homogeneous sets. Note that for any formula $\varphi(\bar{x}, \bar{v})$ there is a first order formula $\bar{H}_{n} \bar{x} \varphi(\bar{x}, \bar{v})$ which is true of $\bar{a}$ just if there is a set $X$ of cardinality $n$ which is homogeneous for $\varphi(\bar{x}, \bar{a})$ but no superset of $X$ is homogeneous for $\varphi(\bar{x}, \bar{a})$.

$M: \quad$ For every $L$-formula $\varphi(\bar{x}, \bar{v})$ there is an $N$ such that if $m>N, T \models \forall \bar{v} \sim \bar{H}_{m} \bar{x} \varphi(\bar{x}, \bar{v})$.

\section{Lemma 3. $M$ implies $E_{0}$.}

Proof. If $E_{0}$ (i.e., $A_{0}$ ) is false then for every $n$ there exists a model $A_{n}$ and a sequence $\bar{a}_{n}$ such that $A_{n} \vDash_{0} H_{n} \bar{x} \varphi\left(\bar{x}, \bar{a}_{n}\right) \wedge \sim Q \bar{x} \varphi\left(\bar{x}, \bar{a}_{n}\right)$. Then for some $m, n<m<\omega A_{n} \vDash_{0} \bar{H}_{m} \bar{x} \varphi\left(\bar{x}, \bar{a}_{n}\right)$ so $M$ fails.

Surprisingly, as we will show later, the converse to this lemma is false.

Definition. A formula $\varphi(\bar{x}, \bar{y})$ has the finite cover property in $T$ if in some model of $T$ for arbitrarily large $n$ there exist $a_{0}, \cdots, a_{n-1}$ such that

$$
A \vDash \bigwedge_{j<n}\left(\exists \bar{x} \bigwedge_{i \neq j} \varphi\left(\bar{x}, \bar{a}_{i}\right)\right) \wedge \sim \exists \bar{x} \bigwedge_{i<n} \varphi\left(\bar{x},{ }_{i} \bar{a}\right) .
$$

The theory $T$ has the finite cover property if some formula $\varphi(\bar{x}, \bar{y})$ has the finite cover property in $T$.

Lemma 4. If $T$ does not have the f.c.p. then $T$ satisfies $M$.

Proof. If $M$ fails then for arbitrarily large $n$, say $n \in J$, there exist $A \models T$ and $\bar{a}_{n}$ in $A$ such that for a fixed formula $\varphi\left(x_{0}, \cdots, x_{m-1}, \bar{v}\right)$, $A \vDash \bar{H}_{n} \bar{x} \varphi\left(\bar{x}, \bar{a}_{n}\right)$. By adding a dummy variable if necessary we may assume $m \geqq 2$. We may assume also that $\varphi$ has the following property: if $x_{2}^{*} \in\left\{x_{0}, \cdots, x_{m-1}\right\}$ for all $i<m$, then $\vDash \varphi(\bar{x}, \bar{v}) \rightarrow \varphi\left(\bar{x}^{*}, \bar{v}\right)$. We will show the formula $\psi\left(x_{0}, \bar{u}\right)=\varphi\left(x_{0}, x_{1}, \cdots, x_{m-1}, \bar{v}\right) \wedge x_{0} \neq x_{1}$ has the f.c.p. Let for each $n \in J, H_{n}=\left\{c_{0}, \cdots, c_{n-1}\right\}$ be a maximal homogeneous set for $\varphi\left(\bar{x}, \bar{a}_{n}\right)$ and let $Z_{n}=\left\{\bar{s}_{0}, \cdots, \bar{s}_{k-1}\right\}$ enumerate the $m-1$ tuples from $H_{n}$. Set, for $i<k, \bar{b}_{i}=\bar{s}_{i} \cap \bar{a}_{n}$. For any $Y \subset k$ we write $P(Y)$ if $A \vDash \exists x_{0} \Lambda_{j \in Y} \psi\left(x_{0}, \bar{b}_{j}\right)$. Then $P(k)$ holds since $A \vDash$ $\forall x_{0}\left[\Lambda_{j<k} \varphi\left(x_{0}, \bar{b}_{j}\right) \rightarrow \mathbf{V}_{l<n} x_{0}=c_{l}\right]$.

Let $V \subset k$ be a minimal set such that $P(V)$ holds. Then $V$ has at least $n$ elements in it, since if $Y \subset k$ has fewer than $n$ elements then $A \models \exists x_{0} \Lambda_{j \in Y} \psi\left(x_{0}, \bar{b}_{j}\right)$ (simply choose $x_{0} \in H_{n}$ but different from the first term of $\bar{b}_{j}$ for all $j \in Y$ ). So listing $V=\left\{\bar{d}_{0}, \cdots, \bar{d}_{l}\right\}$ where 
$l \geqq n-1$ we see that $A \vDash \neg \exists x_{0} \Lambda_{i<l} \psi\left(x_{0}, \bar{d}_{i}\right)$ since $P(V)$ holds, but $A \vDash \Lambda_{j<l} \exists x_{0} \Lambda_{i \neq j} \psi\left(x_{0}, \bar{d}_{i}\right)$ by the minimality of $V$. Thus $\psi\left(x_{0}, \bar{u}\right)$ has the finite cover property.

We collect the preceeding results in:

THEOREM 5. (a) If the complete theory $T$ does not have f.c.p. then $T$ satisfies $E_{0}$.

(b) In particular, if $T$ is $\aleph_{1}$-categorical then $T$ satisfies $E_{0}$.

Proof. Part (a) follows immediately from the lemmas. For (b) we need only recall Keisler's theorem [8] that an $\aleph_{1}$-categorical theory does not have f.c.p.

Now we show that for stable theories $E_{0}$ exactly captures the notion of the f.c.p. We rely on: Theorem A (Shelah [13, II, §4.4]). If $T$ is stable and has the f.c.p. then there is a formula $\varphi\left(x_{1}, x_{2}, \bar{v}\right)$ such that for every $\bar{a}, \varphi\left(x_{1}, x_{2}, \bar{a}\right)$ is an equivalence relation and for arbitrarily large $n$ there exist $\bar{a}_{n}$ and $k, n \leqq k<\omega$, such that $\varphi\left(x_{1}, x_{2}, \bar{a}_{n}\right)$ has exactly $k$ equivalence classes. (This result was obtained independently but later by G. Cooper.)

THEOREM 6. If $T$ is stable, the following properties of $T$ are equivalent: $E_{0}, M$, f.c.p.

Proof. By the lemmas above, it suffices to show that if $T$ has the f.c.p. then $T$ does not have $E_{0}$. Choose $\varphi\left(x_{1}, x_{2}, \bar{v}\right)$ to satify Theorem $\mathrm{A}$, and consider

$$
\varphi^{\prime}\left(x_{1}, x_{2}, \bar{v}\right): x_{1} \neq x_{2} \longrightarrow \sim \varphi\left(x_{1}, x_{2}, \bar{v}\right) .
$$

If $E_{0}$ holds, for some $n$ :

$$
T \vDash{ }_{0} H_{n} x_{1} x_{2} \varphi^{\prime}\left(x_{1}, x_{2}, \bar{v}\right) \longrightarrow Q x_{1}, x_{2} \varphi^{\prime}\left(x_{1}, x_{2}, \bar{v}\right) .
$$

But this contradicts the conclusion of Theorem A.

Note that in Theorem 6 we are able to apply $E_{0}$ to a formula involving $Q^{2}$. Thus for stable theories the eliminability of the $Q^{2}$ quantifier in the $\aleph_{0}$-interpretation implies the eliminability of all the $Q^{n}$. In contrast the language $L\left(Q^{n+1}\right)$ is strictly stronger than the language $L\left(Q^{n}\right)$ (due in the $\aleph_{0}$-interpretation to Shelah [12] and in the $\aleph_{\alpha}$-interpretation for $\alpha>0$ to Shelah [12], Garavaglia [6] and (assuming $\diamond$ ) to Baudisch [2]). Presumably, some hypothesis on the theory $T$ is necessary since Cowles [5] pointed out the theory of real closed field eliminates the quantifier "there exists infinitely many", but does not satisfy $E_{0}$.

We now consider the extent that these results apply to other formalizations of the notion, "for many sequences $\bar{x}, \varphi(\bar{x})$ holds." 
Sticking first to the $\boldsymbol{\aleph}_{0}$-interpretation we introduce the Ramsey quantifier on sequences.

Definition. Let $\bar{x}_{1}, \cdots, \bar{x}_{m}$ be an $m$-tuple of $n$-tuples. Form the logic $L\left(Q_{0}^{*}\right)$ where $A \vDash_{0} Q^{*, m, n} \bar{x}_{1}, \cdots, \bar{x}_{m} \varphi\left(\bar{x}_{1}, \cdots, \bar{x}_{m}\right)$ just if there is an infinite set $Y$ of $n$-tuples from $A$ such that $\bar{y}_{1}, \cdots, \bar{y}_{m} \in Y$ implies $A \models_{0} \varphi\left(\bar{y}_{1}, \cdots, \bar{y}_{m}\right)$.

By applying our earlier arguments to sequences rather than elements one obtains the following.

TheOREM 7. (i) If $T$ is $\boldsymbol{\aleph}_{0}$-categorical or does not have the f.c.p. then $T$ admits elimination of quantifiers in $L\left(Q_{0}^{*}\right)$.

(ii) If $T$ is stable then $T$ admits elimination of quantifiers in $L\left(Q_{0}^{*}\right)$ if and only if $T$ does not have the f.c.p.

The quantifiers $Q_{0}^{*}$ generalize two notions in the literature other than the Ramsey quantifiers.

Definition. (i) $A \models_{0} I^{m} x_{1}, \cdots, x_{m} \varphi\left(x_{1}, \cdots, x_{m}\right)$ if there are infinitely many pairwise distinct sequences $\left\langle\bar{a}_{i}: i\langle\omega\rangle\right.$ such that $A \models_{0} \varphi\left(\bar{x}_{i}\right)$.

(ii) $A \models_{0} I^{*, m} x_{1}, \cdots, x_{m} \varphi\left(x_{1}, \cdots, x_{m}\right)$ if there exist infinitely many $m$-ary sequences $\left\langle\bar{a}_{i}: i\langle\omega\rangle\right.$ such that $A \models_{0} \varphi\left(\bar{a}_{i}\right)$ and if $i \neq j$ then $\operatorname{rng} \bar{a}_{i} \cap \operatorname{rng} \bar{a}_{j}=\varnothing$.

Clearly $I^{m}$ is just the quantifier $Q^{*, m, 1}$, while $I^{*, m} \varphi(x)$ is equivalent to

$$
Q^{*, 2, m} \bar{x}_{1}, \bar{x}_{2}\left[\varphi\left(\bar{x}_{1}\right) \wedge \varphi\left(\bar{x}_{2}\right) \wedge\left[\bigwedge_{j<m} x_{1}^{j}=x_{2}^{j} \vee \bigwedge_{i, j<m} x_{1}^{i} \neq x_{2}^{j}\right]\right]
$$

where $\bar{x}_{1}=\left\langle x_{1}^{0}, \cdots, x_{1}^{m-1}\right\rangle$ and $\bar{x}_{2}=\left\langle x_{2}^{0}, \cdots, x_{2}^{m-1}\right\rangle$.

Schmerl [11] considers a variant of $I^{*}$ and remarks that it is eliminable in a theory which is $\boldsymbol{\aleph}_{0}$-categorical. Winkler [17] proves that $I^{*}$ is eliminable in any theory which is either $\boldsymbol{\psi}_{0}$ or $\boldsymbol{\aleph}_{1}$-categorical. All of these results follow from Theorem 7 .

One sense in which $I$ and $I^{*}$ are weaker than the Ramsey quantifier is that there exists a stable theory $T$ in which both $I$ and $I^{*}$ are eliminable but $T$ does not have the f.c.p.

For this, consider a language with infinitely many constant symbols $c_{k}$ and one ternary relation symbol $E(x, y, z)$. Partition an infinite set $X$ into infinitely many infinite classes $X_{i}$ for $i \in \omega$ and each $X_{i}$ into $i+1$ classes $X_{i j}$ for $j \leqq i$ with each $X_{i j}$ infinite. Now let $E(a, b, c)$ hold just if for some $i a, b, c$ are all in $X_{i}$ and for some $j$ both $a$ and $b$ are in $X_{i j}$. Let the constants name one member of 
$X_{i j}$, for each $i$ and $j$. It is easy to see that the formula $\varphi(x ; y)$ : $E(x, x, y) \wedge \sim E(x, y, y)$ has the finite cover property. On any saturated model of $T$ the maximal quantifier-free types are first order complete so $T$ is quantifier eliminable in $L$. Thus, to show that $T$ admits elimination of the quantifier $I^{*}$, it suffices to find for any quantifier-free $L$-formula $\varphi(\bar{x}, \bar{y})$, an $L$-formula equivalent in $T$ to $I^{*} \bar{x} \varphi(\bar{x}, \bar{y})$. Now if $A \vDash T$ and $A \vDash \exists \bar{x} \varphi(\bar{x}, \bar{a})$ then $A \vDash{ }_{0} I^{*} x \varphi(\bar{x}, \bar{a})$ unless $\varphi(\bar{x}, \bar{y})$ logically implies that some $x_{i}$ is equal to some $y_{j}$ or to constant $c_{k}$ (necessarily occuring in $\varphi$ ). But then $I^{*} \bar{x} \varphi(\bar{x}, \bar{a})$ is equivalent to $\exists x_{1}\left(x_{1} \neq x_{1}\right)$. The quantifier $I$ is handled similarly, replacing "some $x_{i}$ " by "each $x_{i}$ ".

Our results partially extend to the logic $L_{c}^{*}$ where $A \models_{c} Q x \varphi(x)$ is interpreted as: there is a set $X$ with $|X|=|A|$ which is homogeneous for $\varphi$. In this logic we assume $A$ is infinite. In particular if we write $A_{c}, E_{c}$ as the obvious analogs of $A_{c}, E_{0}$ we get by the same proof as Theorem 1. Theorem 1': $E_{c} \Leftrightarrow A_{c}$.

We want to extend Theorem $5 \mathrm{~b}$ to the equicardinality interpretation. The required lemma is

Lemma 8. If $\varphi(x)$ is an L-formula, $A$ is $|A|^{+}$-universal and $A \vDash{ }_{0} Q x \varphi(x)$ then $A \vDash{ }_{c} Q x \varphi(x)$.

Proof. Since $A \models_{0} Q x \varphi(x)$, by compactness and downward Lowenheim-Skolem there is a model $B$ of $T$ with $|B|=|A|$ and $B \vDash{ }_{c} Q x \varphi(x)$. But since $A$ is $|A|^{+}$universal it follows that $A \models_{c} Q x \varphi(x)$.

THEOREM 9. If $T$ is $\aleph_{1}$-categorical then $T$ satisfies $E_{c}$.

Proof. By Theorem 5b, $E_{0}$ and hence $A_{0}$ holds of $T$; we show $A_{c}$ holds of $T$. It suffices to show for any $A T$ and $L$-formula $\varphi$ that $A \models_{0} Q \bar{x} \varphi(\bar{x}, \bar{a})$ implies $A \models_{c} Q \bar{x} \varphi(\bar{x}, \bar{a})$. But this is tautological if $A$ is countable and follows immediately from $\aleph_{1}$-categoricity and Lemma 8 if $A$ is uncountable.

Note that we did not prove that if $A$ is saturated and $\varphi$ is an $L^{*}$-formula then $A \vDash_{0} \varphi$ implies $A \vDash_{c} \varphi$. In fact, that assertion is false as can be seen by examining the saturated model of cardinality $\aleph_{1}$ of the theory of an equivalence relation $E$ with one equivalence class of size $n$ for each finite $n$. The relevant formula is $Q x \sim$ QyE $(x, y)$.

Is there some "first order property" of $T$ which is equivalent to $E_{0}$ ? Clearly, $\sim$ f.c.p. is not equivalent to $E_{0}$ in general since any $\boldsymbol{\aleph}_{0}$-categorical unstable theory has the f.c.p. [14] but also has $E_{0}$ by Theorem 2. A more likely candidate is the condition $M$. The following example dashes this hope. 
THEOREM 10. There is an $\aleph_{0}$-categorical theory $T_{0}$ which does not satisfy $M$.

Proof. Let $L_{0}$ be a language containing one binary relation $R$ and for all $n<\omega, n+1$-ary relations $P_{n}$ and $Q_{n}$. We let $\alpha_{n}\left(x_{0}, \cdots, x_{n}\right)$ denote the formula asserting all the $x_{i}$ are distinct and $R\left(x_{i}, x_{j}\right)$ holds for all $i \leqq j \leqq n$. Let $T_{0}$ be axiomatized by the universal closure of

(i ) $R(x, x) \wedge(R(z, y) \rightarrow R(y, z))$

(ii) $P_{n}(\bar{x}) \rightarrow\left[\alpha_{n}(\bar{x}) \wedge \forall y\left(\Lambda_{i \leq n} y \neq x_{i} \rightarrow \approx \Lambda_{i \leq n} R\left(y, x_{i}\right)\right)\right]$

(iii) $Q_{n}(\bar{x}) \rightarrow \alpha_{n}(\bar{x})$

(iv) $\alpha_{n}(\bar{x}) \rightarrow\left(P_{n}(\bar{x}) \Leftrightarrow \sim Q_{n}(\bar{x})\right)$.

Now $T_{0}$ is a universal theory with the joint embedding and amalgamation properties (the union of any two models is a model), so $T_{0}$ has a countable ultrahomogeneous (i.e., homogeneous in the sense of Jonsson [7]) and universal model $A$. The symbols $P_{n}$ and $Q_{n}$ are trivial on sequences of less than $n$ elements so for each $n$ the number of nonisomorphic substructures of $A$ with cardinality $n$ is finite. Whence by ultrahomogeneity and the Svenonious characterization of $\boldsymbol{\aleph}_{0}$-categoricity, $T=T h(A)$ is $\boldsymbol{\aleph}_{0}$-categorical. Moreover $T$ admits elimination of quantifiers because in the only countable model of $T$ the quantifier free types are complete. Let $A^{*}$ be the reduct of $A$ to the language whose only relational symbol is $R$. The $P_{n}$ and $Q_{n}$ are definable from $R$ in $T$. In particular, $T \vDash P_{n}(\bar{x}) \Leftrightarrow\left(\alpha_{n}(\bar{x}) \wedge \forall y\left[\Lambda_{i \leqq n} y \neq x_{i} \rightarrow\right.\right.$ $\left.\left.\mathrm{V}_{i \leqq n} \sim R\left(y, x_{i}\right)\right]\right)$. This follows from the universality of $A$. Thus $T^{*}=T h\left(A^{*}\right)$ is also $\aleph_{0}$-categorical. But it is easy to see that $A^{*}$ contains arbitrarily large maximal finite homogeneous set for $R(x, y)$, namely sets $\left\{x_{0}, \cdots, x_{n}\right\}$ such that $A \vDash P_{n}(\bar{x})$. Hence $T^{*}$ does not satisfy $M$.

$A$ first order theory $T$ is said to be finitely model complete if there is an extension of $T$ by adding a finite number of definable predicates which is model complete. J. L. Paillet [10] asked whether every $\boldsymbol{\aleph}_{0}$-categorical theory is finitely model complete.

THEOREM 11. There is an $\aleph_{0}$-categorical theory which is not finitely model complete.

Proof. The theory $T^{*}$ defined in the previous theorem provides an example. Since $T$ is quantifier eliminable (and a foritori, model complete), if any finite definitional extension of $T^{*}$ is model complete, for some $n$, the theory $T_{n}$ obtained by adding the symbols $P_{m}, Q_{m}$ $m \leqq n$ and their definitions to $T^{*}$ must be model complete. But no such $T_{n}$ is model complete. Indeed, $P_{n+1}(\bar{x})$ is equivalent in $T_{n}$ to a universal but not to an existential formula.

When the second author suggested the example for Theorem 9, 
the first author recalled its similarity (virtual identity, as it turns out) to the example of $2^{\aleph_{0}} \boldsymbol{\aleph}_{0}$-categorical theories due to Ash [1]. Our verification of the $N_{0}$-categoricity of $T$ is by Ash's method.

There are several further questions suggested by this work.

1. Find a "purely first order" property equivalent to $E_{0}$. One notion of "first order property" is suggested by Cooper in [3]. It may be too restrictive for our purposes. At the other extreme one can ask if $E_{0}$ is an absolute property of $T$.

2. For $T$ a theory in a finite language, if $T$ is $L_{0}^{*}$-complete must it have $E_{0}$ ? This seems more likely if, in addition, $T$ is stable. Matt Kaufman has shown the assumption that $L$ is finite is essential here.

3. Does the theory of differentially closed fields of characteristic $p, p \geqq 0$, satisfy $E_{0}$ ? Equivalently, does each such theory fail to satisfy the f.c.p.?

4. For $T$ a theory in a finite language, if $T$ admits $E_{c}$ must $T$ be $\aleph_{1}$-categorical? This is false if we don't assume the language is finite. Since $E_{c}$ implies $T$ has no two cardinal models the question reduces to, "if $T$, in a finite language, satisfies $E_{c}$ must it be $\omega$ stable?".

Added in Proof. (1) Recall that Shelah [14] has proved that every unstable theory has the f.c.p. Thus Theorem 6 could be rephrased as

THEOREM 6 '. Thas f.c.p. iff $T$ is stable and $E_{0}$ holds. We used the formulation in Theorem 6 because the goal in this paper is the characterisation of $E_{0}$.

(2) The quantifier elimination given by our proofs is not effective (Theorem 2 and Lemma 4 are the relevant ones). In each case we know (either by Ryll-Nardzewski or the failure of f.c.p.) that a certain finite number exists for each formula $\psi(\bar{u})$, but we do not know how to compute it effectively. H. Kierstead and Jeff Remmel have exhibited a complete decidable theory in $L$ which is not decidable in $L^{*}$ even though it is $\omega$-categorical.

(3) P. Tuschik and P. Rothmaler have shown question 4 has a negative answer. Subsequently we verified that the theory of an infinite binary tree with two successor functions is such an example

\section{REFERENCES}

1. Christopher J. Ash, Undecidable $\boldsymbol{\aleph}_{0}$-categorical theories, Notices Amer. Math. Soc., 18 (1971), 423.

2. A. Baudisch, Uncountable $n$-cubes in models of $\boldsymbol{\aleph}_{0}$-categorical theories, (to appear).

3. G. Cooper, On complexity of complete first order theories, (thesis), Simon Fraser, 1979. 
4. J. Cowles, The relative expressive power of some logics extending first order logic, J. Symbolic Logic, 44 (1979), 129-145.

5. - The theory of Archimedean real closed fields in logics with Ramsey quantifiers, (to appear).

6. S. Garavaglia, Relative strength of Malitz quantifiers, (to appear).

7. B. Jonsson, Homogeneous universal relational systems, Math. Scand., 8 (1960), 137142.

8. H. J. Keisler, Ultra products which are not saturated, J. Symbolic Logic, 32 (1967), $23-47$.

9. M. Magidor and J. Malitz, Compact extensions of L(Q), (part la), Ann. Math. Logic, 11 (1977), 217-263.

10. J. L. Paillet, Contributed talk in Nroclaw, Poland, A. S. L. European summer meeting, 1977.

11. J. Schmerl, Decidability and finite axiomatizability of $\boldsymbol{\aleph}_{0}$-categorical partially ordered sets, (to appear).

12. S. Shelah, Letter to Cowles, (1976).

13. Classification Theory and the Number of Nonisomorphic Models, North Holland Publishing Co., Amsterdam, 1978.

14. - Stability the f. c. p., and Superstability; model theoretic properties of formu. las in first order theory, Annals Math. Logic, 3 (1971), 271-362.

15. R. L. Vaught, Denumerable Models of Complete Theories, in Infinitistic Methods, PWN, Warsaw, (1961), 303-321.

16. S. Vinner, Model-completeness in a first order language with a generalized quantifier, Pacific J. Math., 56 (1975), 265-273.

17. P. Winkler, Model completeness and Skolem expansions, Thesis (Yale), 1975.

Received May 18, 1979 and in revised form July 23, 1979. The first author was partially supported by NSF Grant 77-01667. The second author was partially supported by NSF-Grant MCS 77-03993.

UNIVERSITY of ILLINOIS

Chicago, IL 60680 



\section{PACIFIC JOURNAL OF MATHEMATICS}

\section{EDITORS}

DONALD BABBITT (Managing Editor)

University of Galifornia

Los Angeles, California 90024

HUGO RossI

University of Utah

Salt Lake City, UT 84112

C. C. MOORE AND ANDREW OGG

University of California

Berkeley, CA 94720
J. DUGUNDJI

Department of Mathematics University of Southern California Los Angeles, California 90007

R. FINN AND J. MILGRAM Stanford University

Stanford, California 94305

ASSOCIATE EDITORS

E. F. BECKENBACH

B. H. NeumanN

F. WOLF

K. YosHidA

\section{SUPPORTING INSTITUTIONS}

UNIVERSITY OF BRITISH COLUMBIA UNIVERSITY OF SOUTHERN CALIFONIA CALIFORNIA INSTITUTE OF TECHNOLOGY UNIVERSITY OF CALIFORNIA MONTANA STATE UNIVERSITY STANFORD UNIVERSITY UNIVERSITY OF HAWAII UNIVERSITY OF NEVADA, RENO UNIVERSITY OF TOKYO U'NIVERSITY OF UTAH NEW MEXICO STATE UNIVERSITY WASHINGTON STATE UNIVERSITY OREGON STATE UNIVERSITY UNIVERSITY OF OREGON UNIVERSITY OF WASHINGTON 


\section{Pacific Journal of Mathematics}

\section{Vol. 90, No. $1 \quad$ September, 1980}

Shashi Prabha Arya and M. K. Singal, On the locally countable sum

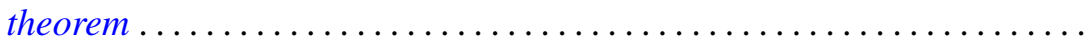

John Theodore Baldwin and David William Kueker, Ramsey quantifiers and

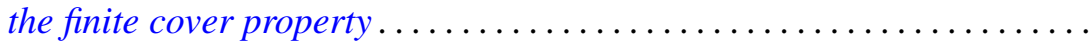

Richard Body and Roy Rene Douglas, Unique factorization of rational

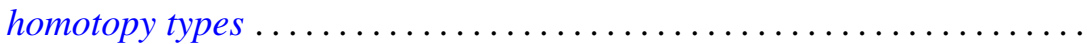

Ethan Bolker and Ben G. Roth, When is a bipartite graph a rigid

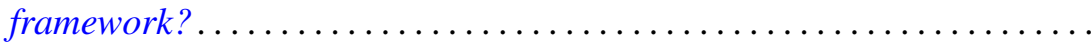

Alicia B. Winslow, Continua in the Stone-Čech remainder of $R^{2} \ldots \ldots \ldots$

Richard D. Carmichael and Elmer Kinji Hayashi, Analytic functions in tubes which are representable by Fourier-Laplace integrals ..............

Stephen D. Cohen, The Galois group of a polynomial with two indeterminate coefficients ..............................

Russell Allan Johnson, Strong liftings commuting with minimal distal

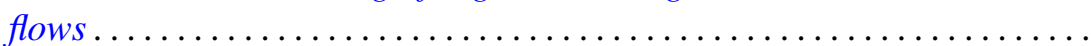

Elgin Harold Johnston, The boundary modulus of continuity of harmonic

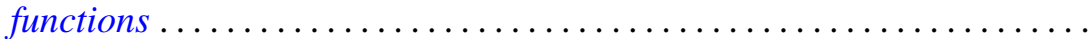

Akio Kawauchi and Takao Matumoto, An estimate of infinite cyclic

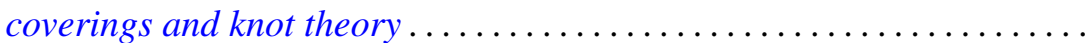

Keith Milo Kendig, Moiré phenomena in algebraic geometry: rational alternations in $\mathbf{R}^{2}$...

Roger T. Lewis and Lynne C. Wright, Comparison and oscillation criteria for selfadjoint vector-matrix differential equations .

Teck Cheong Lim, Asymptotic centers and nonexpansive mappings in conjugate Banach spaces .......................

David John Lutzer and Robert Allen McCoy, Category in function spaces.

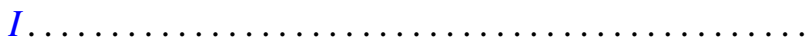

Richard A. Mollin, Induced p-elements in the Schur group ...

Jonathan Simon, Wirtinger approximations and the knot groups of $F^{n}$ in $S^{n+2}$

Robert L. Snider, The zero divisor conjecture for some solvable groups...

H. M. (Hari Mohan) Srivastava, A note on the Konhauser sets of biorthogonal polynomials suggested by the Laguerre polynomials...

Nicholas Th. Varopoulos, A probabilistic proof of the Garnett-Jones theorem on BMO.

Frank Arvey Wattenberg, $[0, \infty]$-valued, translation invariant measures on $N$ and the Dedekind completion of ${ }^{*} R \ldots \ldots \ldots \ldots . .$. 